\title{
Prediction of Polyphenol Oxidase Activity Using Visible Near- Infrared Hyperspectral Imaging on Mushroom (Agaricus bisporus) Caps.
}

\author{
Edurne Gaston \\ Technological University Dublin, edurne.gaston@tudublin.ie \\ Jesus Maria Frias \\ Technological University Dublin, Jesus.Frias@tudublin.ie \\ Patrick Cullen \\ Technological University Dublin, pj.cullen@tudublin.ie
}

See next page for additional authors

Follow this and additional works at: https://arrow.tudublin.ie/schfsehart

Part of the Food Processing Commons

\section{Recommended Citation \\ Gaston E, Frias JM, Cullen PJ, O'Donnell CP, Gowen AA. (2010): Prediction of Polyphenol Oxidase Activity Using Visible Near-Infrared Hyperspectral Imaging on Mushroom (Agaricus bisporus) Caps. Journal of Agricultural and Food Chemistry 58(10) 6226-6233. DOI: 10.1021/jf100501q}

This Article is brought to you for free and open access by the School of Food Science and Environmental Health at ARROW@TU Dublin. It has been accepted for inclusion in Articles by an authorized administrator of ARROW@TU

Dublin. For more information, please contact arrow.admin@tudublin.ie, aisling.coyne@tudublin.ie, gerard.connolly@tudublin.ie.

Funder: Irish Government Department of Agriculture, Fisheries and Food under the Food Institutional Research Measure (FIRM).

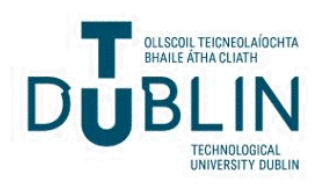




\section{Authors}

Edurne Gaston, Jesus Maria Frias, Patrick Cullen, Colm O'Donnell, and Aoife Gowen 


\section{Prediction of Polyphenol Oxidase Activity Using vis-NIR Hyperspectral Imaging on Mushroom (Agaricus bisporus) Caps}

Running title header: Prediction of PPO using HSI on mushroom caps

EDURNE GASTON ${ }^{1}$, JESÚS M. FRÍAS ${ }^{1 *}$, PATRICK J. CULLEN ${ }^{1}$, COLM P. O'DONNELL $^{2}$ and AOIFE A. GOWEN .

${ }^{1}$ School of Food Science and Environmental Health, Dublin Institute of Technology, Cathal Brugha Street, Dublin 1, Ireland.

${ }^{2}$ Biosystems Engineering, School of Agriculture, Food Science and Veterinary Medicine, University College Dublin, Dublin 4, Ireland.

*Corresponding author. E-mail: Jesus.Frias@dit.ie 
2 Physical stress (i.e. bruising) during harvesting, handling and transportation triggers 3 enzymatic discoloration of mushrooms, a common and detrimental phenomenon largely 4 mediated by polyphenol oxidase (PPO) enzymes. Hyperspectral imaging (HSI) is a non-

5 destructive technique that combines imaging and spectroscopy to obtain information from a

6 sample. The objective of this study was to assess the ability of HSI to predict the activity of

7 PPO on mushroom caps. Hyperspectral images of mushrooms subjected to various damage

8 treatments were taken, followed by enzyme extraction and PPO activity measurement.

9 Principal component regression (PCR) models (each with 3 PCs) built on raw reflectance and 10 multiple scatter corrected (MSC) reflectance data were found to be the best modeling 11 approach. Prediction maps showed that the MSC model allowed for compensation of 12 spectral differences due to sample curvature and surface irregularities. Results reveal the possibility of developing a sensor which could rapidly identify mushrooms with higher

14 likelihood to develop enzymatic browning and hence aid produce management decision 15 makers in the industry.

17 KEYWORDS: polyphenol oxidase, tyrosinase, mushrooms, Agaricus bisporus, vis-NIR hyperspectral imaging. 
Button mushrooms (Agaricus bisporus) production is a fermentation industry that is able to produce quality protein from cellulose based agricultural by-products (1). White button mushrooms are one of the most important horticultural crops grown in Ireland with more than 60,000 tons produced annually (2). This produce is very sensitive to inappropriate handling and transportation practices, which cause irreversible injuries on the mushrooms and enhance cap discoloration (3).

Browning of mushrooms is the major cause of quality loss that accounts for reduction in their market value. Development of browning is the consequence of a series of biochemical reactions in which polyphenol oxidase (PPO) enzymes, naturally present in mushrooms, play an important oxidative role $(4,5)$. The PPO family includes catechol oxidase and laccase, both of which oxidise diphenols into corresponding quinones (6). Quinones are slightly colored products that undergo further reactions leading to high molecular mass dark pigments called melanins. Brown discoloration is largely confined to the skin tissue of the mushroom, where levels of phenols and polyphenol oxidase are higher than in other parts of the fungi (7). PPO inactivation has been the target of several postharvest treatments including thermal or microwave heating (8), irradiation (9) and addition of inhibitors (10). However, consumer preference for fresh produce makes the management of PPO activity a problem in the production, distribution and retail of fresh mushrooms.

Hyperspectral imaging (HSI) is a rapid and non-destructive technology that has recently emerged as a powerful process analytical tool for food analysis (11). Hyperspectral images are composed of hundreds of contiguous wavebands for each spatial position of an object. 
position. Hyperspectral images, known as hypercubes, are three-dimensional blocks of data, comprising two spatial and one wavelength dimension. Hypercube classification enables the identification of regions with similar spectral characteristics. Since regions of a sample with similar spectral properties have similar chemical composition, hypercube classification allows for the visualisation of biochemical constituents of an object, as well as their concentration and distribution over the sample. Due to the large size of hypercubes, multivariate analytical tools, such as stepwise multiple linear regression (MLR), principal component regression (PCR) and partial least squares regression (PLSR) are usually employed for hyperspectral data mining and identification of key wavelengths for the development of automated multispectral sensors.

Rapid spectroscopic techniques show potential for replacement of slow and/or expensive analytical measurements while retaining sufficient accuracy (12). Recent studies have demonstrated $\mathrm{HSI}$ to be a useful technology for the investigation of various mushroom quality related issues, such as deterioration (13), freeze damage detection (14) and blemish characterization (15). Recent advances in the application of HSI to the assessment of safety and quality of other foodstuffs also include contaminant detection $(16,17)$, defect identification (18-20), constituent analysis (21) and quality evaluation (22-24).

So far, hyperspectral imaging has not been employed to study the activity of enzymes in mushrooms. Short wavelength infrared hyperspectral imaging was recently used to predict $\alpha$-amylase activity at early germination stages in two classes of wheat kernels and $R^{2}$ values of 0.54 and 0.73 , respectively, were achieved (25). Given that polyphenol oxidases play a key role in the mushroom browning process and that extraction and current activity measurement techniques, such as radiometric, electrometric, chronometric and especially 
spectrophometric (26), are time consuming (as an example, in this study, 1.5-2 hours were needed to obtain an extract and measure its activity), it would be desirable to have a fast and non-destructive system that could estimate enzyme activity on mushroom caps. The development of a hyperspectral imaging system with the ability to make simultaneous predictions on multiple mushroom caps could enable faster detection of produce likely to lose market value and hence reduce economical losses in the industry.

The aim of the present study was to investigate the potential of vis-NIR (445-945 nm) hyperspectral imaging for the prediction of PPO enzyme activity on mushroom caps.

\section{MATERIAL AND METHODS}

\section{Mushroom supply and damage}

Agaricus bisporus mushrooms (strain Sylvan A15, Sylvan Spawn Ltd., Peterbourough, UK) were grown in plastic bags and tunnels in Kinsealy Teagasc Research Centre (Kinsealy, Co. Dublin, Ireland) following common practice in the mushroom industry. Only uniform undamaged closed cap mushrooms from the $1^{\text {st }}$ and $2^{\text {nd }}$ flush with a diameter of $3-5 \mathrm{~cm}$ were hand-picked, placed in a metal grid and carefully delivered to the laboratory in purpose-built containers, to minimize mechanical damage during transport. Mushrooms arrived at the laboratory premises within 1 hour after harvesting and were stored overnight at $4^{\circ} \mathrm{C}$.

Some samples were subjected to vibrational bruising to simulate crop handling and transport. Mushrooms were damaged in batches of 600g (approx) units inside polystyrene plastic boxes. Mechanical damage was induced by using a Gyratory Shaker Model G2 
shaking table (New Brunswick scientific Co., Edison, N.J., USA) at $300 \mathrm{rpm}$ amplitude for controlled periods of time. A shaking period of $10 \mathrm{~min}$ led to loss of 6 units of lightness $\left(\mathrm{L}^{*}\right)$ and color difference $(\Delta E)$ of 7.79 in C.I.E. L ${ }^{*} a^{*} b^{*}$ color space. A shaking period of 20 min led to loss of 12 units of $\mathrm{L}^{*}$ and $\Delta \mathrm{E}$ of 15.57. $\Delta \mathrm{E}$ defines the magnitude of the total color difference and is expressed by the following equation:

$$
\Delta E=\sqrt{L_{0}^{*}-L^{* 2}+a_{0}^{*}-a^{*^{2}}+b_{0}^{*}-b^{* 2}}
$$

where the ${ }_{0}$ subscript refers to color measurements before shaking and no subscript refers to color measurements after shaking.

Mushrooms were placed on polystyrene trays in groups of approx. 10 and over-wrapped with PVC film following common practice in the mushroom industry. The trays were stored under refrigeration (GRAM K4OOLU, Denmark) for the duration of the experiment.

Mushrooms of three damage levels [undamaged (D0), 10 min shaking damage (D10) and 20 min shaking damage (D20)] were monitored throughout five time points (days 0, 1, 2, 3 and 6 of storage).

At each sampling time point during refrigerated storage, one tray of each damage level was randomly selected and removed from storage 15 min prior to testing. Wrapping was removed and all the mushrooms in the packet were scanned with the hyperspectral imaging equipment, then subsequently divided into two groups of five mushrooms for enzyme extraction. This procedure was repeated for each tray. A total number of 549 mushrooms were scanned and 114 extracts were obtained. 
Hyperspectral images were obtained using a pushbroom line-scanning HSI instrument (DV

111 Optics Ltd, Padua, Italy). The instrument comprised a moving table, illumination source (150

112 W halogen lamp source attached to a fiber optic line light positioned parallel to the moving table), mirror, objective lens (16 mm focal length), Specim V10E spectrograph (Spectral

114 Imaging Ltd, Oulu, Finland) operating in the wavelength range of 400-1000 nm 115 (spectroscopic resolution of $5 \mathrm{~nm}$ ), CCD camera (Basler A312f, effective resolution of $580 \times$ 116580 pixels by 12 bits), acquisition software (SpectralScanner, DV Optics, Padua, Italy) and PC.

117 A cylindrical diffuser was placed in front of the fiber optic line light to produce a diffuse light source. In this study, only spectral data within the wavelength range of 445-945 $\mathrm{nm}$ were used, as beyond this range the noise level of the camera is high and the signal efficiency of the light source is low.

\section{$\underline{\text { Reflectance calibration }}$}

Reflectance calibration was carried out prior to mushroom image acquisition in order to account for the background spectral response of the instrument and the "dark" camera response. The bright response ('W') was obtained by collecting a hypercube from a uniform white ceramic tile; the dark response ('dark') was acquired by turning off the light source, completely covering the lens with its cap and recording the camera response. The corrected reflectance value ('R') was calculated from the measured signal ('I') on a pixel-by-pixel basis as shown by:

$$
R_{i}=\frac{\left(I_{i}-\operatorname{dark}_{i}\right)}{\left(W_{i}-\operatorname{dark}_{i}\right)}
$$


where $i$ is the pixel index, i.e. $i=1,2,3, \ldots, n$ and $n$ is the total number of pixels.

Mushroom homogenates were prepared in duplicate from each sample tray, as follows:

$5 \mathrm{~g}$ of the outer skin of mushroom caps were extracted using a sharp knife, chopped and placed in a Turrax homogenizer (ULTRA-TURRAX T25, Janke \& Kunkel IKA Labortechnik, Germany) in a 1:4 (w: v) ratio with $0.5 \mathrm{M}$ phosphate buffer, $\mathrm{pH} 6.5$, containing $50 \mathrm{~g} / \mathrm{L}$ polyvinylpirrolidone (Sigma-Aldrich, Dublin, Ireland). Homogenization was carried out for 1 $\min$ at $4^{\circ} \mathrm{C}$ and $8000 \mathrm{rpm}$. The homogenate was centrifuged (2K15 Laborzentrifugen, SIGMA, Germany) at $12,000 \mathrm{~g}$ for $35 \mathrm{~min}$ at $4^{\circ} \mathrm{C}$. The supernatant was collected by filtration through no. 1 Whatman paper and used as crude enzyme extract. Extracts were kept at $4^{\circ} \mathrm{C}$ in the dark until spectrophotometric assay (within $2 \mathrm{~h}$ ).

PPO activity was measured spectrophotometrically by a modified method based on those of

Galeazzi et al. (27) and Tan and Harris (28). The reaction mixture contained $0.1 \mathrm{~mL}$ crude enzyme extract and $2.9 \mathrm{~mL}$ substrate solution [0.011 mol/L catechol (Sigma-Aldrich, Dublin, Ireland) as substrate in $0.05 \mathrm{~mol} / \mathrm{L}$ phosphate buffer, $\mathrm{pH}$ 6.5]. The rate of catechol oxidation was followed at $410 \mathrm{~nm}$ (UV2 UV/vis Spectrometer, UNICAM, UK) and $25^{\circ} \mathrm{C}$ and represented against time. The maximum slope of the straight-line section of the activity curve was used to express the enzyme activity (EAU/g of fresh mushroom). A unit of enzyme activity was defined as an increase of 0.001 absorbance units per minute.

Enzyme activity was measured in triplicate for each mushroom extract and the average value was computed. The standard error (SE) of this method was $350.50 \mathrm{EAU} / \mathrm{g}$ of fresh mushroom. 
Data were recorded in reflectance, saved in ENVI header format using the acquisition software and then exported to MATLAB R2007b (The Math Works, Inc. USA).

\section{$\underline{\text { Masking }}$}

A masking step was carried out to separate the mushroom pixels from the background. The mask was created by thresholding the mushroom image at $940 \mathrm{~nm}$, where a pixel threshold value of 0.2 was used to segment the mushroom from the background. All background regions were set to zero and the non-zero elements of the image were used to extract one mean spectrum for each mushroom.

\section{$\underline{\text { False RGB images }}$}

False RGB images were obtained by extracting mushroom images at $460 \mathrm{~nm}$ (blue), $545 \mathrm{~nm}$ (green) and $645 \mathrm{~nm}$ (red) and stacking them.

165 One of the main challenges involved in building predictive models with hyperspectral image data is that such images contain a vast amount of spectral data, whilst only one or a few measurements of the variable of interest can be taken for each sample studied. In this particular study, the reference method for enzyme extraction involved using the skin of three to five mushrooms to obtain one single enzyme extract. Consequently, three to five hyperspectral images were to be matched with one single enzyme activity value in regression modeling. 
When developing regression models with hyperspectral data, it is common practice to extract the mean spectrum of each sample and use it to build a prediction model to estimate an attribute (29). With that approach in mind, two different modeling strategies were used:

a) Strategy 1: The first strategy extracted the mean spectrum of each mushroom and assigned the same enzyme activity value to all the mushrooms used in obtaining one particular extract. A training set of $n_{\text {TRAIN } 1}=280$ and a test set of $n_{\text {TEST_1 }}=269$ were used for this strategy.

b) Strategy 2: The second strategy computed the mean spectra of all the mushrooms used to obtain one enzyme extract and assigned the enzyme activity value of that extract to the resulting spectrum. A training set of $n_{\text {TRAIN_2 }}=60$ and a test set of $\mathrm{n}_{\text {TEST_2}}=54$ were used for this strategy.

The following spectral preprocessing methods were used in order to remove non-chemical biases, such as scattering effects and variations arising from mushroom surface curvature, from the spectral information: standard normal variate (SNV) (30) and multiplicative scatter correction (MSC) (31). MSC aims to reduce the effects of scattering in a set of spectra by performing linear regression on a "target" spectrum. Two different target spectra led to two different MSC methods: a) "set MSC", where the mean spectrum of each mushroom was corrected using the mean spectrum of the data set as the target spectrum and b) "sample $\mathrm{MSC}^{\prime \prime}$, where the spectrum of each pixel in a mushroom was corrected using the mean spectrum of that mushroom as the target spectrum. The mean sample MSC corrected spectrum for each mushroom was obtained and used for the model. 
To improve normality of the distribution of the reference variable, enzyme activity values were transformed into natural logarithmic units and mean centered.

a) Multiple linear regression (MLR): optimal wavelengths for enzyme activity prediction were selected by the "forward" method in best subsets stepwise linear regression using the "leaps" package in R (32). Multicolinearity of predictor variables is problematic for MLR models based directly on spectroscopic values, tending to results in unstable model predictions (33). The variance inflation factor (VIF) is an index commonly used to measure the colinearity between variables in regression models: typically, predictor variables with VIF $>10$ are considered to be highly correlated. In order to test the predictor wavelengths for multicolinearity, the VIF of each predictor was calculated using the "DAAG" package in $\mathrm{R}(32)$.

b) Principal component regression (PCR): principal component analysis (PCA) reduces the dimensionality of spectral data by transforming them into principal component scores in order of decreasing variance. The autoscaled matrix of spectral values was transformed into PC space by representing the original data in the directions defined by orthogonal eigenvectors using R (32). PCR models were developed using PC space scores instead of wavelength space values. Analysis of variance (ANOVA) was employed using R (32) to compare models with increasing number of PCs. The decision on the number of PCs to be taken for each model was made based upon ANOVA test results. Only significant components $(p<0.05)$ were included in the model. 
c) Partial least squares regression (PLSR): this technique is commonly used when predicting a response from many measured variables which may be collinear. PLSR was applied using the "pls" package in R (32). Leave-one-out cross-validation was used on the training set. Performance of the prediction models was evaluated using the root of the mean of the sum of squared differences between predicted and measured enzyme activity values of the training set (RMSECV) and the number of latent variables required (\# LV). The optimal number of latent variables for inclusion in the PLSR models was estimated using the method described by Martens et al. (34)

The experiment was carried out two times, making two independent mushroom sets: a training set $\left(\mathrm{n}_{\text {TRAIN_1 }}=280\right.$ mushrooms and $\mathrm{n}_{\text {TRAIN_2 }}=60$ extracts $)$ and a test set $\left(\mathrm{n}_{\text {TEST_1 }}=269\right.$ mushrooms and $\mathrm{n}_{\text {TEST_2 }}=54$ extract). Overall, 549 mushrooms were used to obtain 114 extracts in total. All of the models were built on training sets and then applied to independent test sets of samples. The ratio of percentage deviation (RPD), which is the ratio of the standard deviation of the laboratory measured (reference) data to the rootmean-square of cross-validation (RPD $\mathrm{DRAIN}_{\text {) }}$ ) or root-mean-square error of prediction (RPD $\mathrm{TEST}_{\text {T }}$ ) (35), was used to assess model performance. Twenty four models were classified in terms of their ability to generalize following criteria outlined by Viscarra Rossel et al. (36), based on which $\mathrm{RPD}_{\mathrm{TEST}}<1.0$ indicates very poor model/predictions and their use is not recommended; $1.0<\mathrm{RPD}_{\mathrm{TEST}}<1.4$ indicates poor model/predictions where only high and low values are distinguishable; $1.4<\mathrm{RPD}_{\mathrm{TEST}}<1.8$ indicates fair model/predictions that may be used for assessment and correlation; $1.8<\mathrm{RPD}_{\mathrm{TEST}}<2.0$ indicates good models/predictions where quantitative predictions are possible; $2.00<R P D_{T E S T}<2.5$ indicates very good, quantitative model/predictions and RPD TEST $_{2.5}$ indicates excellent model/predictions. 
240 The two models whose performance was found to be best were selected and applied to

241 each pixel in the hypercube data of individual mushrooms. This enabled the generation of

242 virtual prediction images for enzyme activity.

RESULTS AND DISCUSSION

\section{Spectra}

246 Average reflectance spectra obtained from the hyperspectral imaging data of undamaged

247 (D0), damaged 10 (D10) and damaged 20 (D20) mushrooms are shown in Figure 1a. The 248 average reflectance of damaged samples was lower than the average reflectance of nondamaged mushrooms over the entire spectral region. Bruising due to mechanical damage was expected to have led to loss of whiteness and lightness $\left(L^{*}\right)$ and therefore lower reflectance values. A remarkable difference in intensity was observed between D0 and D20 mushrooms, whereas the intensity of D10 spectra was intermediate between D0 and D20. Broad spectra in the visible-near infrared wavelength range are characteristic of undamaged mushrooms, corresponding to their white appearance (13). The greatest differences in shape between bruised and non-bruised samples arose in the $600-800 \mathrm{~nm}$ region, where undamaged mushrooms exhibited broader spectral features than the damaged mushrooms.

257 The spectral differences mentioned above could be related to the formation of brown pigments (14) mainly melanins, which derive from enzyme-catalyzed oxidation products called quinones. 
261 The average polyphenol oxidase enzyme activity of each mushroom group is shown in

262 Figure $1 \mathrm{~b}$. The higher activity values observed in bruised mushrooms suggest that mechanical damage has an effect on enzyme expression. Considering that physical injuries are one of the factors that lead to mushroom browning (3) and that this phenomenon is mediated by PPO enzymes (37), this result was not unexpected. The difference in PPO activity between D10 and D20 was not significant ( $p>0.05)$, which could mean that the stress caused by D10 damage level was sufficiently high to bring enzyme expression to its maximum, and further damage did not contribute to further activation of tyrosinase.

\section{Modeling}

VIF was greater than 10 for every MLR model built with more than two wavelengths.

271 Therefore, MLR models that used only two wavelengths were considered for further analysis. In the case of PCR models, the inclusion of the third PC was not always significant $(p<0.05)$ so 2 and 3 PC models were considered for further sections. For all PLSR models, 2 was the optimal number of latent variables to include in the model. Previous studies in the field employed models that performed well using low numbers of wavelengths (13),

276 principal components $(14,38)$ or PLS latent variables (39).

277 Model performance in terms of RPD is shown in Table 1. RPD TRAIN $_{\text {in }}$ a measure of model performance within the model training data set and RPD

279 performed when applied to an independent model testing data set. RPD ${ }_{\text {TEST }}$ was considered 280 to be more adequate to assess model performance and further sections of this paper will

281 focus only on RPD TEST values. 
282 Models were classified in terms of RPD ${ }_{\text {TEST }}$ as follows: RPD $_{\text {TEST }}<1.0=$ "very poor", $1.0<\mathrm{RPD}_{\mathrm{TEST}}<1.4=$ "poor"; $1.4<\mathrm{RPD}_{\mathrm{TEST}}<1.8=$ "fair"; $1.8<\mathrm{RPD}_{\mathrm{TEST}}<2.0=$ "good"; $2.00<R^{2} D_{T E S T}<2.5=$ "very good" and $\mathrm{RPD}_{\mathrm{TEST}}>2.5=$ "excellent".

\section{Strategy}

Overall, models with a better generalization ability to predict the independent data set were and chemometric technique combination, the RPD obtained under model strategy 1 (i.e. when the mean spectrum of each mushroom was extracted and the same enzyme activity value was assigned to all the mushrooms used for one extract) was higher than the RPD obtained under model strategy 2 (i.e. when the mean spectra of all the mushrooms used to obtain one enzyme extract was computed and the enzyme activity value of that extract was assigned to the resulting spectrum). In fact, strategy 2 only gave "poor" or "very poor" predictive models, whose RPD ${ }_{\text {TEST }}$ ranged from 0.81 to 1.3 . This could be because when the mean spectrum was computed for an extract under strategy 2 , some features arising from the original spectral variability of the mushrooms within that extract might have been lost. This would result in partial loss of their ability to generalize and decrease in RPD

\section{Pre-treatment}

For MLR, raw reflectance spectral data and sample MSC corrected reflectance spectra led to better performance models than SNV or set MSC spectra. The better models were "fair" and the worse ones were "poor" (according to the previously mentioned RPD classification) and therefore discarded. Similar trends were observed in PCR models, where "very good" models were obtained with raw reflectance and sample MSC corrected reflectance spectra 
$\left(\mathrm{RPD}_{\mathrm{TEST}}=2.13\right.$ with $\left.3 \mathrm{PCS}\right)$, a "good" model with SNV pre-treated reflectance data $\left(R P D_{\text {TEST }}=1.84\right.$ with $\left.2 \mathrm{PCS}\right)$ and a "fair" model with set MSC corrected reflectance spectra 306 (RPD $_{\text {TEST }}=1.77$ with 2 PCS). The number of PCs was lower in the case of SNV and set MSC but adding a third one did not significantly improve model performance or RPD $\mathrm{TEST}_{\text {. For }}$ PLSR models, all pre-treatments resulted in "poor" models, whose highest RPD $\mathrm{TEST}_{\text {Was }}$ 1.22.

\section{$\underline{\text { Regression method }}$}

Under strategy 1, PCR models performed better than MLR or PLSR models for all of the pretreatments. This happened for both training and test sets. The performance of MLR and PLSR models for the test set was not as good as it was for the training set, but that did not happen for PCR models, where RPD $D_{\text {TEST }}$ values were higher than RPD ${ }_{\text {TRAIN }}$ values.

Under model strategy 2, all chemometric methods performed similarly for the training set. For the test set, PCR models performed better than MLR or PLSR but still "poor" predictions $\left(\mathrm{RPD}_{\mathrm{TEST}}<1.3\right)$ were obtained.

PCR models developed on raw reflectance and sample MSC corrected reflectance data under model strategy 1 were selected as best models and used in further analysis. The coefficient of determination and root mean-squared error of cross-validation/prediction for these models were: $\mathrm{R}_{\text {TRAIN_1 }}^{2}=0.75, \mathrm{RMSECV}=0.38[\ln (\mathrm{EAU} / \mathrm{g})], \mathrm{R}_{\text {TEST_}}^{2}=0.78$ and RMSEP $=0.30$ [In(EAU/g)]. Root mean-squared errors of cross-validation/prediction are frequently used to assess the performance of the regression and low values indicate good models.

In Figure 2, enzyme activity values predicted by one of the selected models (model strategy 1, PCR, raw reflectance data) are plotted against experimental enzyme activity values, for (a) training and (b) test sets, respectively. The range of measured reference values was wider in 
the training set than in the test set, where PPO activity levels were, in general terms, lower and confined to a narrower range of values. This scenario is not optimal for model testing but it is common when dealing with horticultural products, whose postharvest behavior is known to be affected by biological variation. Burton (3) reported that mushroom bruisability can vary from crop to crop. A study by Mohapatra et al. (40) observed $30 \%$ to $41 \%$ variability in enzyme activity measurements and attributed it to batch-to-batch variability. Some vertical scattering can be seen in this figure too, indicating variability in predicted values for mushrooms with similar reference enzymes activities. This would explain the relatively low values of the coefficients of determination obtained $\left(R_{\text {TRAIN_1 }}^{2}=0.75\right.$ and $\left.R_{\text {TEST_1 }}^{2}=0.78\right)$. The horizontal scattering is mainly attributable to mushroom to mushroom variability.

\section{Prediction maps}

Hyperspectral imaging has the ability to map the spatial distribution of components on a sample. The two selected models (model strategy 1, PCR, non-treated reflectance and sample MSC corrected reflectance) were applied to each pixel in the hypercube data of individual mushrooms and that enabled the generation of virtual prediction images for enzyme activity. In such images, the grayscale intensity is related to the value of the predicted enzyme activity at different regions of the mushroom cap: the lighter the color, the higher the predicted activity value.

Figure 3 and Figure 4 show the predicted distribution of enzyme activity over the cap of undamaged (D0) and damaged (D20) mushroom samples, respectively. Each figure shows (a) false RGB images, (b) prediction maps based on the raw reflectance model and (c) prediction maps based on the sample MSC pre-treated reflectance model of four mushroom caps whose skin was processed together to obtain one single enzyme extract. The mean and 
standard deviation (SD) of the predictions, both in $[\ln (E A U / g)]$, are displayed below each map in (b) and (c). The values below false RGB images correspond to the activity measurement obtained experimentally for each extract, which is the same for all of the mushrooms within each figure.

The main difference between the prediction images of D0 and D20 is the grayscale intensity. The dark gray tonality in Figures $3(\mathrm{~b})$ and $3(\mathrm{c})$ indicates that the models predicted low activity values on D0 mushroom caps. D20 predictions, on the contrary, show much lighter colours in Figures 4(b) and 4(c), which reveal higher predicted values for enzyme activity. At scanning time, damaged mushrooms looked different from undamaged ones and the corresponding extracts exhibited much higher enzyme activity, for which it was expected that the models would generate very different prediction images according to damage level.

For all of the mushrooms in Figures 3 and 4, the mean predicted values by raw reflectance and sample MSC corrected reflectance models (displayed under each image in columns (b) and (c)) were very similar. This indicates that both raw reflectance and sample MSC corrected reflectance models performed very similarly in terms of quantitative prediction. This is in agreement with the similarities observed previously in the coefficient of determination and the root-mean-square error of both models. However, the very different appearance of predictions maps in (b) and (c) point out these two models have some dissimilarities too.

- In raw reflectance predicted images (Figures $3(\mathrm{~b})$ and $4(\mathrm{~b})$ ), the distribution of enzyme activity prediction is uneven throughout the cap. The relatively high standard deviation values under each map reveal this heterogeneity too. As clearly seen in Figure 3(b), the highest predicted values concentrate around the mushroom 
edges, (i.e the region showing higher level of bruising on false RGB images (Figure 3(a)). This could be partly due to increased presence of brown coloured pigments at edge regions, which are derived from PPO-mediated reaction products, but spectral differences related to mushroom curvature might have also affected the performance of the model differently in different regions of the cap. It is difficult to estimate the extent of such phenomena and at this point. The lack of shading effects in Figure 4(b), where predicted values do not show any clear morphological trend, suggest that the effect of sample curvature on the reflectance model may not be observable when the levels of damage and browning are high.

- However, it is interesting to note that all of $3(b)$ and $4(b)$ figures reveal the ability of this model to point out the regions that look "different" in false RGB images. The model captures the spectral variability arising from surface bruises/marks (e.g. confined regions which show browner colour in false RGB images) and reflects it onto the prediction maps. For undamaged mushrooms, Figure 3(b) exhibits lighter grayscale tonality (indicating higher predicted value) on the small regions that show signs of brusing in Figure 3(a). Similarly, for damaged mushrooms, Figure 4(b) presents darker color (indicating lower predicted value) on those regions where browning had yet not developed in Figure 4(a).

- Sample MSC corrected reflectance predicted images, on the other hand, appear smoother than raw reflectance predictions. All the pixels within one sample MSC corrected reflectance prediction image have similar predicted values and therefore the grayscale intensity is very uniform and the SD values are low. The MSC correction estimates the relation of the scatter of each pixel with respect to the target spectrum (in this case, the mean spectrum of all the pixels) (31). Thus, a similar level 
of scatter is obtained for all spectra and the effect that the morphology of the sample (i.e. mushroom curvature) could have on the model is diminished too.

Figure 5 shows the enzyme activity prediction of imaginary lines drawn through the centre of each mushroom cap, shown in red in Figure 5(a). Figure 5(b) shows how the raw reflectance model predicted the pixel values on those lines; the pixels that form the line are represented in the $X$ axes, while the predicted enzyme activity values are shown in the $Y$ axes. The line in Figure 5(c) corresponds to the prediction of the sample MSC corrected reflectance model. For an undamaged mushroom (see top row), the curved shape of the prediction line in (b) indicates that pixels from the centre and edge regions of the cap were predicted differently; the activity was low in the central region of the mushroom and increased gradually towards the edges. This is in agreement what was observed in Figure 3(b) and could be because the enzyme activity distribution was not uniform along the mushroom cap surface or because this model is not able to deal with spectral differences arising from mushroom cap surface curvature. The line in (c), predicted by the sample MSC corrected reflectance model, is much flatter than the one in (b), which indicates that predictions along the imaginary line were more homogeneous and suggests enzyme activity was equally distributed over the mushroom cap. Despite the fact that both models predicted similar mean activity values $(9.91[\ln (\mathrm{EAU} / \mathrm{g})]$ and $9.94[\ln (\mathrm{EAU} / \mathrm{g})]$, respectively), differences in pixel distribution suggest that the ability of each model to overcome spectral variability due to sample morphology is different. For damaged mushrooms (see bottom row), the line predicted by the reflectance model (b) was uneven but, as opposed to what 417 was observed in the undamaged mushroom, it did not have a clear curved shape. In this case, the variation of predicted enzyme activity values across the imaginary line could be 
419 related to the level of damage/browning, whereas the relationship between predicted 420 values and pixel position/surface curvature was not as clear as for undamaged mushrooms.

421 The line in (c) was flatter than in (b), as observed for undamaged mushrooms. Raw 422 reflectance and and sample MSC corrected reflectance models predicted almost identical 423 mean enzyme activity values $(10.36[\ln ($ EAU/g)] and $10.37[\ln ($ EAU/g) $]$, respectively) and 424 their distributions across pixel line was more similar than in the case of undamaged 425 mushrooms.

426 The ability of a HSI system to predict PPO activity on mushroom caps was assessed in this 427 study. PPO activity prediction maps of were generated to gain understanding of (a) the 428 distribution of the enzyme activity over the mushroom cap and (b) the effect of sample MSC 429 pre-treatment on the predictive ability of the model. Results reveal some potential of vis430 NIR hyperspectral imaging as a tool to estimate the activity of enzymes responsible for 431 mushroom browning. The mushroom industry could benefit from such a tool for rapid 432 identification of mushrooms of reduced marketability. 


\section{FIGURES}
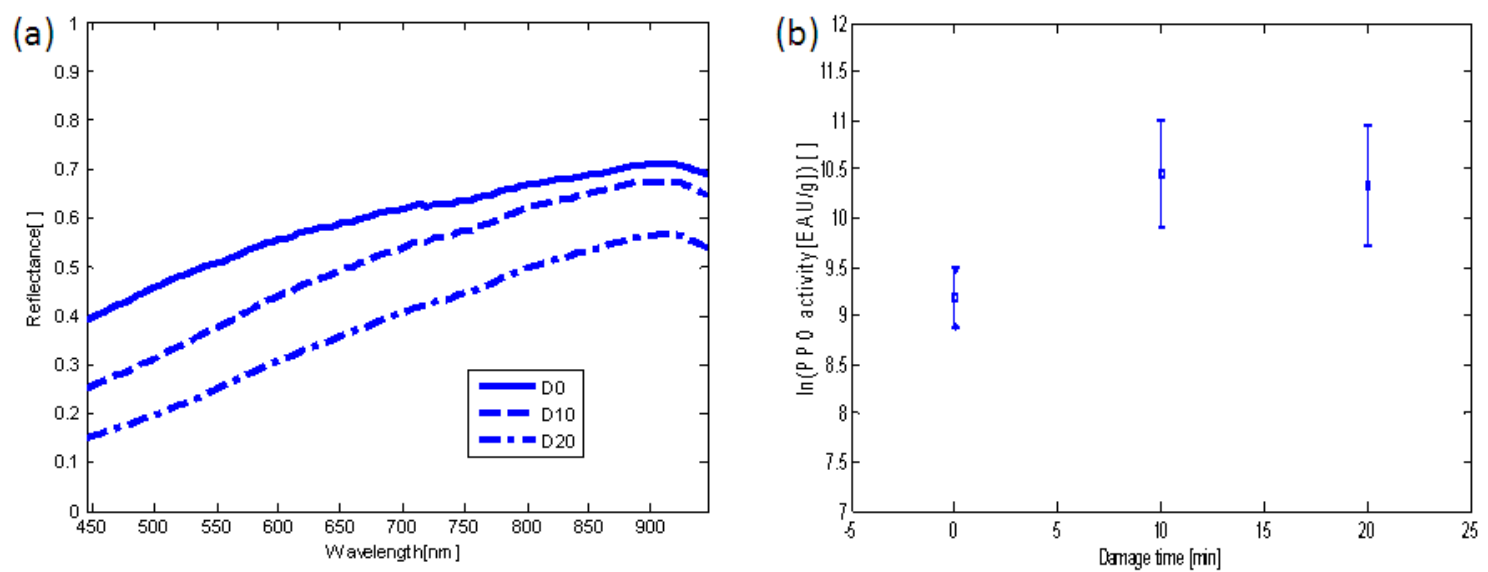

Figure 1 (a) Average raw reflectance spectra for mushroom at different damage levels. (b) Average \pm standard deviation of polyphenol oxidase activity as a function of damage level.

(a)

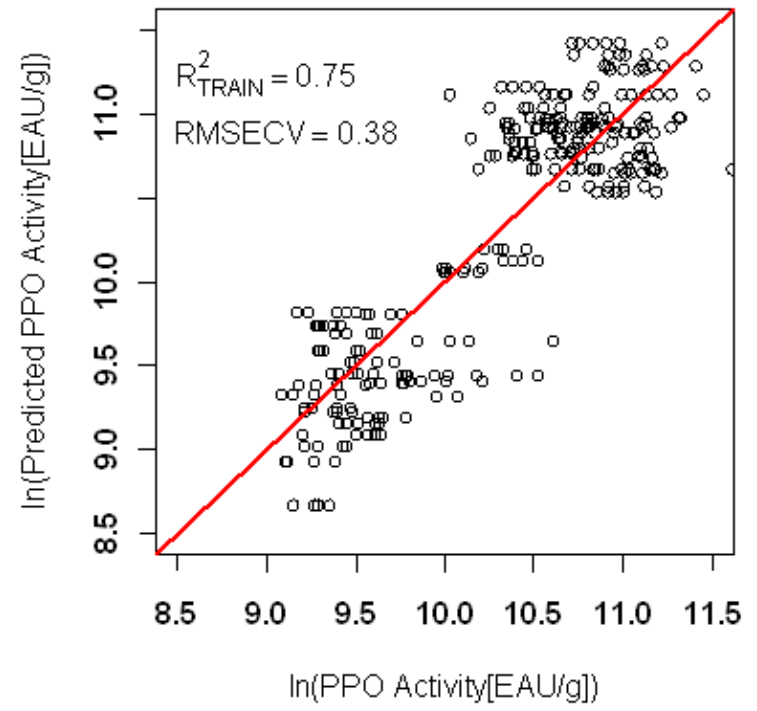

(b)

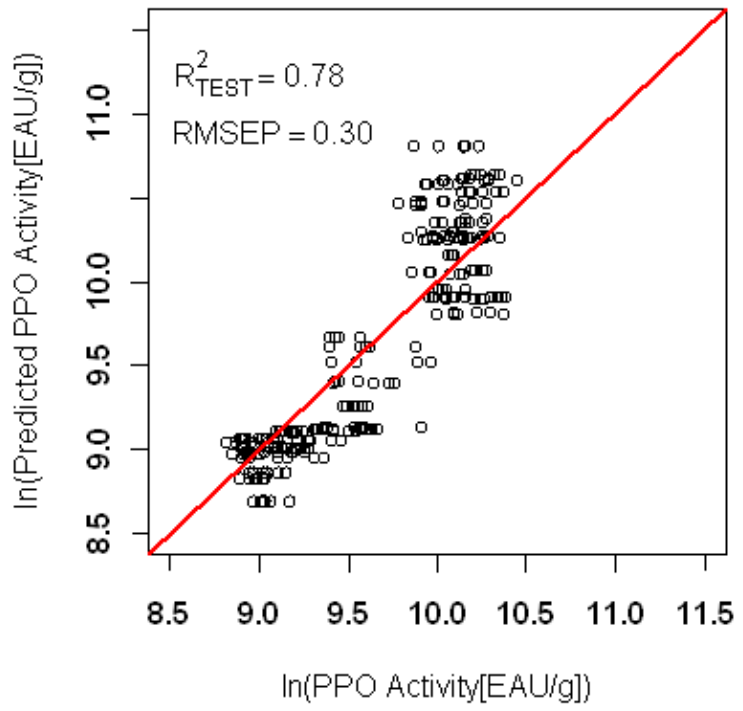

Figure 2 Predicted PPO activity as a function of actual PPO activity for 3 PC PCR model applied to training (left) and test (right) raw data sets under model strategy 1. 
(a)

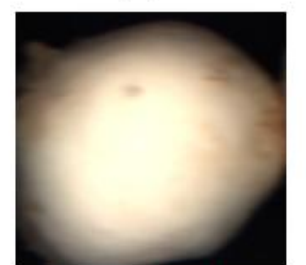

Measured $=9.33$
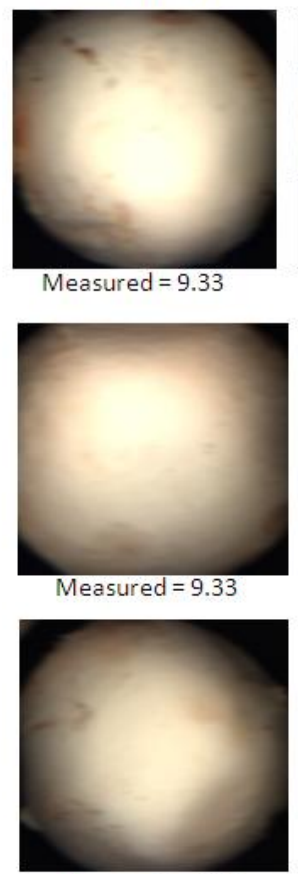

Measured $=9.33$ (b)

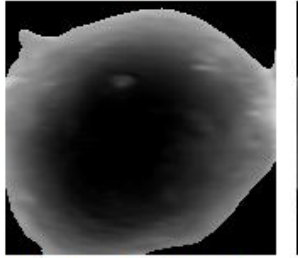

Mean $=9.08 ; S D=0.85$

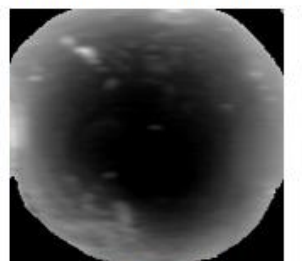

Mean $=9.15 ; \widehat{S D}=0.81$
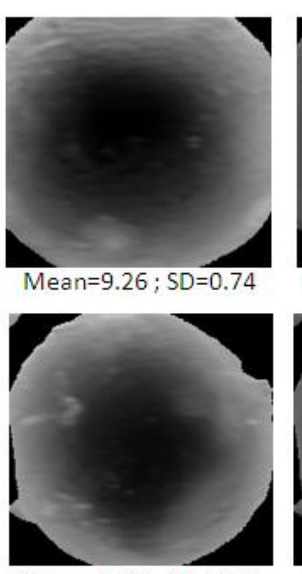

Mean $=9.41 ; \mathrm{SD}=0.73$ (c)

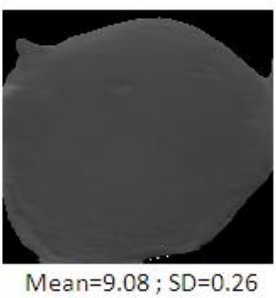

; $S D=0.26$
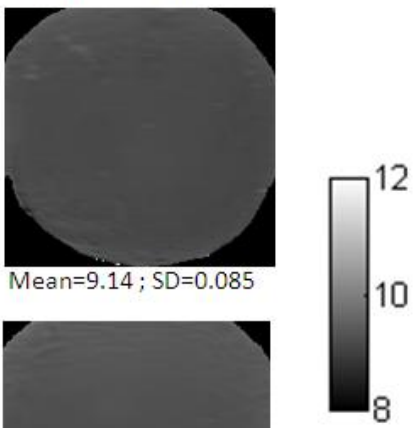

Figure 3 Undamaged mushroom caps, where (a) false RGB image, (b) prediction maps by raw reflectance model and (c) prediction maps by sample MSC corrected reflectance model. (a)

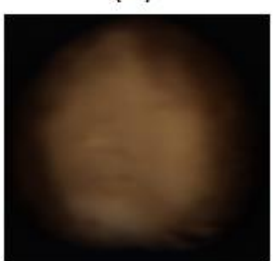

Measured $=10.98$

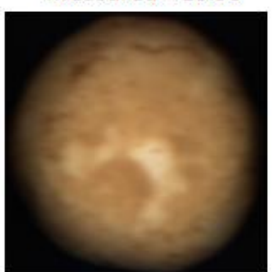

Measured $=10.98$

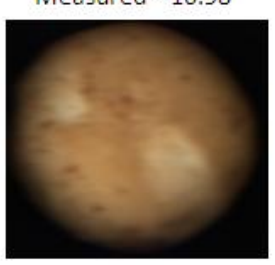

Measured $=10.98$

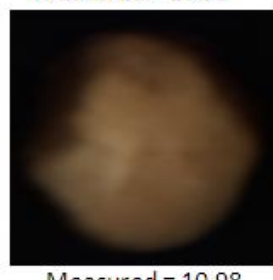

Measured $=10.98$ (b)

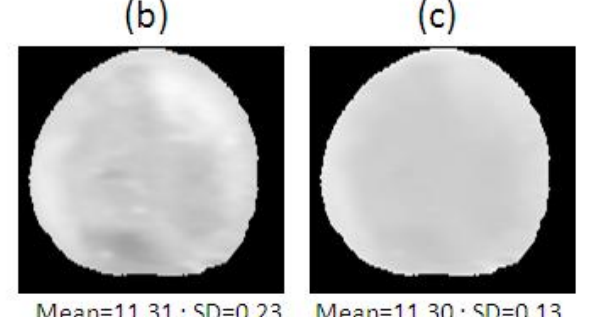

Mean $=11.31 ; S D=0.23$ Mean $=11.30 ; S D=0.13$

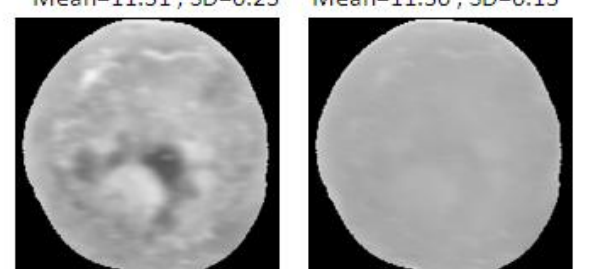

Mean $=10.76 \cdot S D=0.32$ Mean $=10.72 ; S D=0.13$

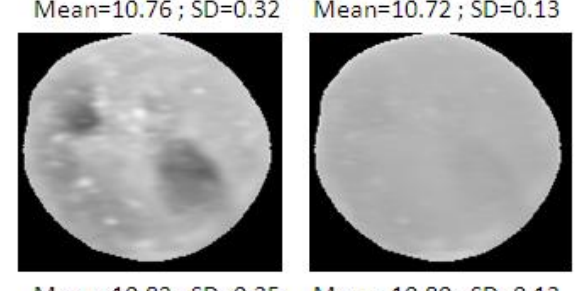

Mean $=10.82 ; S D=0.35 \quad$ Mean $=10.80 ; S D=0.13$

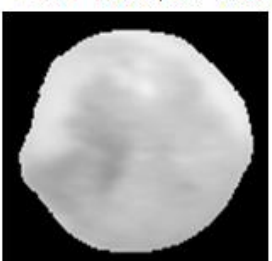

Mean=11.13;SD=0.30 Mean=11.11; SD=0.14

Figure 4 Damaged mushroom caps, where (a) false RGB images, (b) prediction maps by raw reflectance model and (c) prediction maps by sample MSC corrected reflectance model. 
(a)

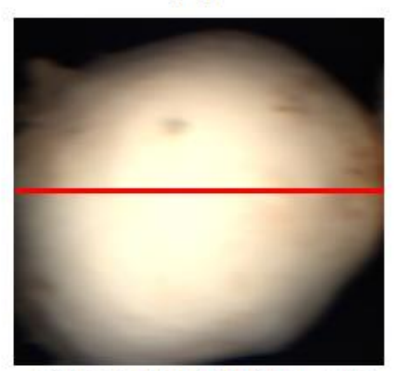

$\ln ($ PPO Activity $[$ EAU/g $])=9.33$

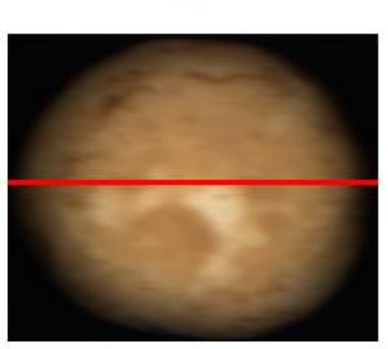

$\ln (\mathrm{PPO}$ Activity $[\mathrm{EAU} / \mathrm{g}])=10.98$ (b)
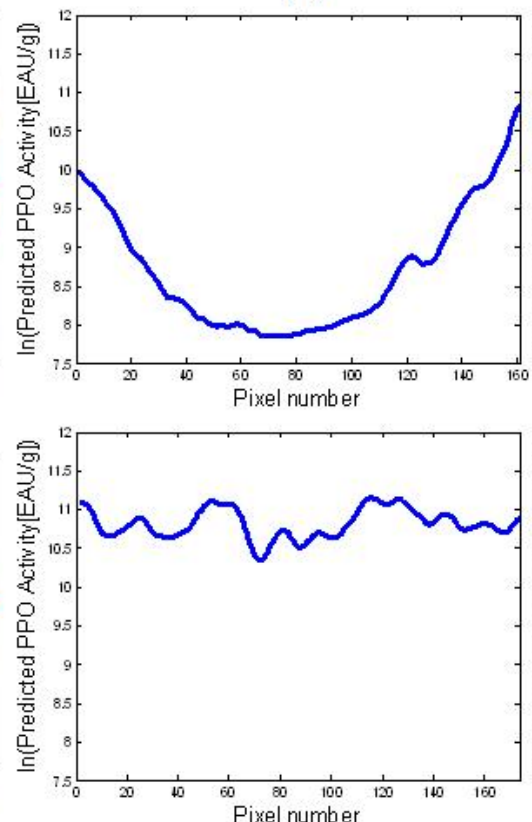

(c)
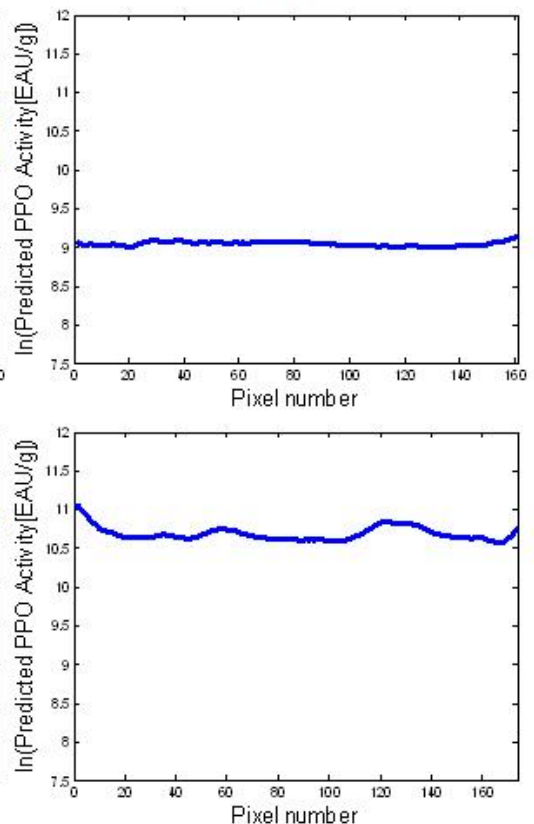

Figure 5 (a) Imaginary line drawn through the centre of false RGB images of undamaged (top row) and damaged (bottom row) mushroom caps and their corresponding predictions by (b) raw reflectance model and (c) sample MSC corrected reflectance model. 


\section{TABLES}

Table 1 Ratio percentage deviation (RPD) for different model strategies, spectral pre-treatments and chemometric methods. MLR: multiple linear regression; PCR: principal component regression; PLSR: partial least squares regression; SNV: standard normal variate; MSC: multiple scatter correction; \# PCs: number of principal components; \# LVs: number of latent variables.

\begin{tabular}{|c|c|c|c|c|c|c|c|c|c|c|}
\hline \multirow[b]{2}{*}{ Strategy } & \multirow[b]{2}{*}{$\begin{array}{l}\text { Pre- } \\
\text { treatment }\end{array}$} & \multicolumn{3}{|c|}{ MLR } & \multicolumn{3}{|c|}{ PCR } & \multicolumn{3}{|c|}{ PLSR } \\
\hline & & $\lambda(\mathrm{nm})$ & RPD $_{\text {TRAIN }}$ & $\mathrm{RPD}_{\text {TEST }}$ & \# PCs & RPD $_{\text {TRAIN }}$ & RPD $_{\text {TEST }}$ & \# LVs & RPD $_{\text {TRAIN }}$ & RPD $_{\text {TEST }}$ \\
\hline \multirow{4}{*}{$1^{*}$} & None & 450,945 & 1.87 & 1.47 & 3 & 2.01 & 2.13 & 2 & 1.95 & 1.16 \\
\hline & SNV & 835,560 & 1.02 & 1.06 & 2 & 1.71 & 1.84 & 2 & 1.63 & 1.22 \\
\hline & Set MSC & 835,545 & 1.52 & 1.14 & 2 & 1.65 & 1.77 & 2 & 1.62 & 1.20 \\
\hline & Sample MSC & 465,945 & 1.91 & 1.43 & 3 & 2.01 & 2.13 & 2 & 1.95 & 1.14 \\
\hline \multirow{4}{*}{$2^{*}$} & None & 470,945 & 1.28 & 1.16 & 2 & 1.27 & 1.30 & 2 & 1.25 & 0.97 \\
\hline & SNV & 450,465 & 1.22 & 1.07 & 1 & 1.17 & 1.20 & 2 & 1.17 & 0.85 \\
\hline & Set MSC & 450,575 & 1.15 & 0.89 & 1 & 1.17 & 1.16 & 2 & 1.17 & 0.81 \\
\hline & Sample MSC & 495,945 & 1.35 & 1.22 & 2 & 1.35 & 1.27 & 2 & 1.33 & 1.22 \\
\hline
\end{tabular}

*as described in Model building subsection of Materials and Methods section. 


\section{ACKOWLEDGEMENTS}

The authors would like to thank Dr. Helen Grogan and Mr. Ted Cormican from Teagasc

Research Station at Kinsealy, Dublin, for provision of mushrooms and kind technical advice.

\section{REFERENCES}

1. Marshall, E.; Nair, N. G. T. Make money by growing mushrooms. Rural Infrastructure and Agro-Industries Division, Food and Agriculture Organization of the United Nations: Rome, 2009; Vol. Diversification booklet number 7 .

2. Teagasc. The irish agriculture and food development authority. Teagasc Mushroom Newsletter 2007, 29.

3. Burton, K. S., Cultural factors affecting mushroom quality - cause and control of bruising. In Science and cultivation of edible and medicinal fungi, Romaine; Keil; Rinker; Royse, Eds. Pennsylvania State University: 2004; pp 397-402.

4. Jolivet, S.; Arpin, N.; Wichers, H. J.; Pellom, G. Agaricus bisporus browning: A review. Mycological Research 1998, 102 (12), 1459-1483.

5. Bandyopadhyay, P.; Jha, S.; Imran Ali, S. K. Picolyl alkyl amines as novel tyrosinase inhibitors: Influence of hydrophobicity and substitution. Journal of Agricultural and Food Chemistry 2009, 57 (20), 9780-9786.

6. Kertesz, D.; Zito, R. Kinetic studies of the polyphenoloxidase action; kinetics in the presence of reducing agents. The indirect oxidation of reduced cytochrome $c$ by polyphenol oxidase. Biochimica et Biophysica Acta 1962, 64, 153-167.

7. Burton, K. S. The effects of pre and post-harvest development on mushroom tyrosinase. Journal of Horticultural Science 1988, 63, 255-260.

8. Devece, C.; Rodriguez-Lopez, J. N.; Fenoll, L. G.; Tudela, J.; Catala, J. M.; de los Reyes, E.; Garcia-Canovas, F. Enzyme inactivation analysis for industrial blanching applications: Comparison of microwave, conventional, and combination heat treatments on mushroom polyphenoloxidase activity. Journal of Agricultural and Food Chemistry 1999, 47 (11), 4506-4511.

9. Beaulieu, M.; D'Apran, M. B. G.; Lacroix, M. Dose rate effect of $\gamma$ irradiation on phenolic compounds, polyphenol oxidase, and browning of mushrooms (Agaricus bisporus). Journal of Agricultural and Food Chemistry 1999, 47 (7), 2537-2543.

10. Weemaes, C. A.; Ludikhuyze, L. R.; Van den Broeck, I.; Hendrickx, M. E. Influence of ph, benzoic acid, glutathione, edta, 4-hexylresorcinol, and sodium chloride on the pressure inactivation kinetics of mushroom polyphenol oxidase. Journal of Agricultural and Food Chemistry 1999, 47 (9), 3526-3530.

11. Gowen, A. A.; O'Donnell, C. P.; Cullen, P. J.; Downey, G.; Frías, J. M. Hyperspectral imaging an emerging process analytical tool for food quality and safety control. Trends in Food Science and Technology 2007, 18, 590-598.

12. Zeaiter, M.; Roger, J. M.; Bellon-Maurel, V. Robustness of models developed by multivariate calibration. Part ii: The influence of pre-processing methods. Trac-Trends in Analytical Chemistry 2005, 24 (5), 437-445.

13. Gowen, A. A.; O'Donnell, C. P.; Taghizadeh, M.; Gaston, E.; O'Gorman, A.; Cullen, P. J.; Frías, J. M.; Esquerre, C.; Downey, G. Hyperspectral imaging for the investigation of quality deterioration in 
sliced mushrooms (Agaricus bisporus) during storage. Sensing and Instrumentation for Food Quality and Safety 2008, 2 (3), 133-143.

14. Gowen, A. A.; Taghizadeh, M.; O'Donnell, C. P. Identification of mushrooms subjected to freeze damage using hyperspectral imaging. Journal of Food Engineering 2009, 93 (1), 7-12.

15. Gowen, A. A.; O'Donnell, C. P.; Taghizadeh, M.; Cullen, P. J.; Frias, J. M.; Downey, G. In Characterisation of blemishes on white mushroom (Agaricus bisporus) caps using hyperspectral imaging. 10th International conference on Engineering and Food - ICEF 10, Viña del Mar, Chile, 2008. 16. Park, B.; Windham, W. R.; Lawrence, K. C.; Smith, D. Contaminant classification of poultry hyperspectral imagery using a spectral angle mapper algorithm. Biosystems Engineering 2007, 96 (3), 323-333.

17. Nakariyakul, S.; Casasent, D. P. Hyperspectral waveband selection for contaminant detection on poultry carcasses. Optical Engineering 2008, 47 (8).

18. Ariana, D. P.; Lu, R. Detection of internal defect in pickling cucumbers using hyperspectral transmittance imaging. Transactions of the ASABE 2008, 51 (2), 705-713.

19. Ariana, D. P.; Lu, R.; Guyer, D. E. Near-infrared hyperspectral reflectance imaging for detection of bruises on pickling cucumbers. Computers and Electronics in Agriculture 2006, 53 (1), 60-70.

20. ElMasry, G.; Wang, N.; Vigneault, C.; Qiao, J.; ElSayed, A. Early detection of apple bruises on different background colors using hyperspectral imaging. LWT - Food Science and Technology 2008, 41 (2), 337-345.

21. Zhao, J. W.; Vittayapadung, S.; Chen, Q. S.; Chaitep, S.; Chuaviroj, R. Nondestructive measurement of sugar content of apple using hyperspectral imaging technique. Maejo International Journal of Science and Technology 2009, 3 (1), 130-142.

22. Qiao, J.; Ngadi, M.; Wang, N.; Gariépy, C.; Prasher, S. Pork quality and marbling level assessment using a hyperspectral imaging system. Journal of Food Engineering 2007, 83 (1), 10-16.

23. Noh, H.; Lu, R. Hyperspectral laser-induced fluorescence imaging for assessing apple fruit quality. Postharvest Biology and Technology 2007, 43, 193-201.

24. ElMasry, G.; Wang, N.; ElSayed, A.; Ngadi, M. Hyperspectral imaging for the nondestructive determination of some quality attributes for strawberry. Journal of Food Engineering 2007, 81 (2), 98-107.

25. Xing, J.; Hung, P. V.; Symons, S.; Shahin, M.; Hatcher, D. Using a short wavelength infrared (swir) hyperspectral imaging system to predict alpha amylase activity in individual Canadian western wheat kernels. Sensing and Instrumentation for Food Quality and Safety 2009, 3, 211-218.

26. Falguera, V.; Pagán, J.; Ibarz, A. A kinetic model describing melanin formation by means of mushroom tyrosinase. Food Research International 2010, 43 (1), 66-69.

27. Galeazzi, M. A.; Sgarbieri, V. C.; Constantinides, S. M. Isolation, purification and physicochemical characterization of polyphenoloxidases (ppo) from dwarf variety of banana (Musa cavendishii). Journal of Food Science 1981, 46, 150-155.

28. Tan, B. K.; Harris, N. D. Maillard products inhibit apple polyphenoloxidase. Food Chemistry 1995, 53, 267-273.

29. Burger, J.; Geladi, P. Hyperspectral NIR image regression part ii: Dataset preprocessing diagnostics. Journal of Chemometrics 2006, 20, 106-119.

30. Barnes, R. J.; Dhanoa, M. S.; Lister, S. J. Standard normal variate transformation and detrending of near infrared diffuse reflectance spectroscopy. Applied Spectroscopy 1989, 43 (5), $772-$ 785.

31. Geladi, P.; MacDougall, D.; Martens, H. Linearization and scatter-correction for near-infrared reflectance spectra of meat. Applied Spectroscopy 1985, 39 (3), 491-500.

32. R_Development_Core_Team $R$ : A language and environment for statistical computing, UK, 2007.

33. Fekedulegn, B. D.; Colbert, J. J.; R.R. Hicks, J.; Schuckers, M. E., Coping with multicollinearity: An example on application of principal components regression in dendroecology. In U.S. Department 
of Agriculture, F. S., Northeastern Research Station (Research Paper NE-721), Ed. USDA Forest Service Newton Square PA: 2002; p 43.

34. Martens, H. A.; Dardenne, P. Validation and verification of regression in small data sets. Chemometrics and Intelligent Laboratory Systems 1998, 44 (1-2), 99-121.

35. Williams, P. C., Variables affecting near-infrared reflectance spectroscopic analysis. In Nearinfrared technology in the agricultural and food industries, Williams, P.; Norris, K., Eds. 1987; pp 143166.

36. Viscarra-Rossel, R. A.; Taylor, H. J.; McBratney, A. B. Multivariate calibration of hyperspectral gamma-ray energy spectra for proximal soil sensing. European Journal of Soil Science 2007, 58, 343353.

37. Mayer, A. M. Polyphenol oxidases in plants and fungi: Going places? A review. Phytochemistry 2006, 67 (21), 2318-2331.

38. Gowen, A. A.; O'Donnell, C. P.; Taghizadeh, M.; Cullen, P. J.; Downey, G. Hyperspectral imaging combined with principal component analysis for bruise damage detection on white mushrooms (Agaricus bisporus). Journal of Chemometrics 2008, 22 (3-4), 259-267.

39. Esquerre, C.; Gowen, A. A.; O'Donnell, C. P.; Downey, G. Initial studies on the quantitation of bruise damage and freshness in mushrooms using visible-near-infrared spectroscopy. Journal of Agricultural and Food Chemistry 2009, 57, 1903-1907.

40. Mohapatra, D.; Frías, J. M.; Oliveira, F. A. R.; Bira, Z. M.; Kerry, J. Development and validation of a model to predict enzymatic activity during storage of cultivated mushrooms (Agaricus bisporus spp). Journal of Food Engineering 2008, 86 (1), 39-48.

This study was funded by the Irish Government Department of Agriculture, Fisheries and Food under the Food Institutional Research Measure (FIRM). 